\title{
Association of Neutrophil to Lymphocyte Ratio with Disease Severity and Joint Effusion in Patients with Early Stage Knee Osteoarthritis
}

\author{
MARIUS IONITESCU1 ${ }^{1}$, DINU VERMESAN ${ }^{1}$, OANA SUCIU ${ }^{2 *}$, BOGDAN DELEANU1, \\ AHMED ABU-AWWAD ${ }^{1}$, VICTOR CRISTIAN DUMITRASCU ${ }^{3}$, ROMEO NEGREA ${ }^{4}$, \\ DAN FRUJA ${ }^{5}$ \\ ${ }^{1}$ Victor Babes University of Medicine and Pharmacy, Department of Orthopedics and Trauma, 2 Eftimie Murgu Sq., \\ 300723, Timisoara, Romania \\ ${ }^{2}$ Victor Babes University of Medicine and Pharmacy, Department of Rehabilitation, Physical Medicine and Rheumatology, \\ 2 Eftimie Murgu Sq., 300723, Timisoara, Romania \\ ${ }^{3}$ Victor Babes University of Medicine and Pharmacy, 2 Eftimie Murgu Sq., 300723, Timisoara, Romania, \\ ${ }^{4}$ Politehnica University of Timisoara, Department of Mathematics, 2 Victoriei Sq., 300723, Timisoara, Romania \\ ${ }^{5}$ West University Vasile Goldis, Faculty of Medicine, 86 Liviu Rebreanu Str, 310414, Arad, Romania
}

\begin{abstract}
The aim of the article was to determine the association between neutrophil to lymphocyteratio (NLR), clinical disease severity and joint effusion in patients with early knee osteoarthritis. We evaluated 79 patients with mild knee degeneration. We obtained NLR from routine hematology panels. As controls, 1260 hematology panels were also obtained. Clinical severity was evaluated from patient reported outcome scores: International Knee Documentation Committee Subjective Knee Evaluation Form, Knee Disability And Osteoarthritis Outcome Score For Joint Replacement, Tegner-Lysholm scale and Euroqol EQ-5D-5L questionnaire. Joint effusion was determined by ultrasound. For the statistics software $R$, version 3.4.4 was used. There were differences in demographics but not in NLR between the study patients and controls (2.03 versus $1.98, p=0.606)$. There was no correlation between NLR and joint effusion $(r 2=-0.121)$ or between NLR, joint effusion and any other parameter recorded ( $r 2$ from - 0.134 to 0.190). In the multiple regression model, NLR was minimally influenced by age $(p=0.059)$ and KOOSJR $(p=0.023)$. Neutrophil to lymphocyte ratio is within normal limits in patients with early stage knee osteoarthritis and is not associated with clinical severity or with joint effusion.
\end{abstract}

Keywords: knee joint, osteoarthritis, ultrasonography, neutrophil to lymphocyte ratio, patient reported outcome measures

\section{Introduction}

Osteoarthritis (OA) is one of the most prevalent diseases in the world, and the knee is the most affected large joint [1]. Diagnosis is mostly based on clinical presentation. Patient reported outcomes (PROs) are the most common measure for quantification [2].

Recent evidence showed that degenerative changes may begin many years before evident radiographic changes. The mechanisms of degeneration are not fully understood but inflammation is incriminated in the pathogenesis of OA. Macrophages and proinflammatory cytokines like IL-6, IL8, IL-1, and TNF- $\alpha$ are known as part of this process [3]. Most of these cytokines are used as biological markers detecting OA but they are used for scientific investigations. There is a need for a simple, inexpensive laboratory test for clinical practice. Inflammation and knee effusion are correlated. Knee effusion and synovial hypertrophy are two of the most common manifestations of knee inflammation and they can be easily determined by ultrasound [4,5]. There is an increased interest in using biomarkers for osteoarthritis to determine early disease, and to identify risk of progression [6]. Neutrophil to lymphocyte ratio (NLR) is a nonspecific marker of inflammation.

*email: oanasuciu78@umft.ro 
It has been proven as a negative outcome predictor in gastrointestinal cancers, cardiovascular events $[7,8]$. However, studies on fractures, OA and inflammatory arthritis did not bring consistent results [9-14].

The aim of our study was to determine the association between NLR, clinical disease severity and joint effusion in patients with early stage knee OA.

\section{Materials and methods}

We included 79 subjects with atraumatic knee pain examined in our department, many of which underwent arthroscopy according to current clinical practice guidelines [15,16]. Incipient degenerative changes (OA) were observed from clinical presentation, imaging studies (magnetic resonance imaging, MRI) and available operative reports $[1,2,15]$.

NLR was determined as the ratio between the absolute number of neutrophils and lymphocytes obtained from routine hematology panels retrieved from the hospital's electronic records [8,9]. Tests were determined using Nihon Kohden Celltac 6500, Sysmex XT-4000i or ADVIA 2120 analyzers. For the control group, all standard hematology panels coded as routine control for hospital employees were retrieved anonymously from the electronic medical records over a period of 2 years.

Patients were evaluated using the international knee documentation committee subjective knee evaluation form (IKDC), the knee disability and osteoarthritis outcome score for joint replacement (KOOSJR), Tegner-Lysholm scale and Euroqol EQ-5D-5L Index (converted using the UK tariff) and visual analogue scale (VAS) $[2,17,18]$.

Joint effusion has been evaluated by ultrasound according to previously reported recommendations $[4,5]$. The knee was examined in approximately 30 degrees of flexion (patient supine on with a cloth rolled under the examined knee). A $12 \mathrm{MHz}$ linear transducer (GE Healthcare, Venue 40) was placed longitudinally over the quadriceps tendon. Effusion was defined as a hypo/anechoic, displaceable and compressible collection in the suprapatellar recess [4, 5]. Effusion size was determined as an average of 2 screens captures as shown in Figure 1. Results were recorded as three categories, as follows: " 0 " if no effusion was present, " 1 " if there was an effusion of $0-4 \mathrm{~mm}$ thickness and " 2 " if the effusion thickness was over $4 \mathrm{~mm}$.

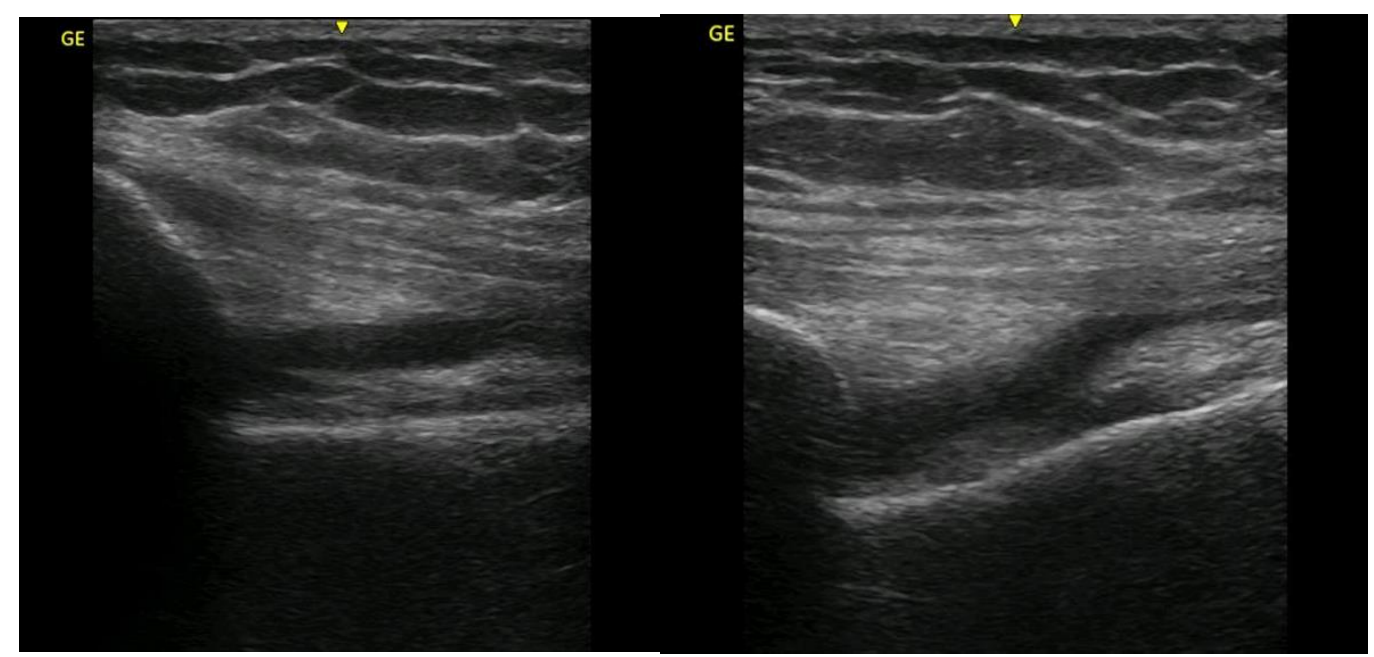

Figure 1. Ultrasound determination of suprapatellar effusion (thickness of $8 \mathrm{~mm}$, category 2)

The study protocol was approved by the Local ethics committee for scientific research and was conducted in accordance with the Declaration of Helsinki.

For the statistics software R, version 3.4.4 was used, with a significance level for alpha being 0.001. Unpaired t-test was used to determine the difference between two means. The backward selection procedure was used to optimize a multiple linear regression model with respond (dependent) 
variable NLR, for all sets of data using R version 3.5.3 statistical software (https://www.r-project.org). Predictor variables were age, gender, Tegner-Lysholm scoring scale, IKDC, effusion, KOOSJR and EQ-5D Index and VAS.

\section{Results and discussions}

There were significant differences in demographics but not in NLR between the study patients and controls: age 54.1 versus 42.4 years old $(\mathrm{p}<0.001)$; M:F ratio 1:1.8 versus 1:5.2 ( $<<0.001)$; average NLR 2.03 versus 1.98 ( $\mathrm{p}=0.606)$. There were 15 patients with no effusion (category 0$), 52$ with category 1 effusion and 12 with significant joint fluid (category 2). The control group consisted of 1260 subjects: $83.96 \%$ females, average age 42.4 years, (range 18-78). In Table 1 higher values of Tegner-Lysholm score,VAS and IKDC score indicate better health. Lower value of KOOSJR score indicate better health. Higher values of NLR (neutrophil to lymphocyte ratio) indicate more inflammation.

Table 1. Demographics and mean values \pm SD of scores

(Tegner -Lysholm, VAS, IKDC, NLR, KOOSJR, effusion)

\begin{tabular}{|c|c|}
\hline Age & $54.1 \pm 11.6$ \\
\hline M:F ratio & $1: 1.8$ \\
\hline Tegner-Lysholm & $46.8 \pm 18.89$ \\
\hline IKDC & $31.9 \pm 14.4$ \\
\hline Effusion & $0.96 \pm 0.58$ \\
\hline KOOSJR & $15.6 \pm 4.78$ \\
\hline Index & $0.51 \pm 0.26$ \\
\hline VAS & $63.4 \pm 19.5$ \\
\hline NLR & $2.03 \pm 0.81$ \\
\hline
\end{tabular}

Correlations between NLR and Age, Gender, Tegner-Lysholm knee rating scale, IKDC, Effusion, KOOSJR, EQ-5D-5L Index and VAS are presented in Table 2 with $p<0.01$

There was no correlation between NLR and joint effusion nor between NLR, joint effusion and any other parameter recorded in Table 2.

In the multiple regression model, NLR of patients with mild knee degeneration was only minimally influenced by age $(\mathrm{p}=0.059)$ and $\operatorname{KOOSJR}(\mathrm{p}=0.023)$.

Table 2. Correlation matrix

\begin{tabular}{|c|c|c|c|c|c|c|c|c|c|}
\hline & Age & Gender & Tegner & IKDC & Effusion & KOOSJR & Index & VAS & NLR \\
\hline Age & 1.000 & -0.132 & -0.332 & -0.290 & -0.010 & 0.372 & -0.211 & -0.179 & -0.124 \\
\hline Gender & -0.132 & 1.000 & 0.188 & 0.307 & 0.138 & -0.342 & 0.259 & 0.412 & 0.066 \\
\hline Tegner & -0.332 & 0.188 & 1.000 & 0.527 & -0.068 & -0.500 & 0.530 & 0.476 & -0.090 \\
\hline IKDC & -0.290 & 0.307 & 0.527 & 1.000 & 0.102 & -0.631 & 0.635 & 0.433 & -0.134 \\
\hline Effusion & -0.010 & 0.138 & -0.068 & 0.102 & 1.000 & 0.017 & 0.066 & 0.061 & -0.121 \\
\hline KOOSJR & 0.372 & -0.342 & -0.500 & -0.631 & 0.017 & 1.000 & -0.457 & -0.483 & 0.190 \\
\hline Index & -0.211 & 0.259 & 0.530 & 0.635 & 0.066 & -0.457 & 1.000 & 0.573 & -0.031 \\
\hline VAS & -0.179 & 0.412 & 0.476 & 0.433 & 0.061 & -0.483 & 0.573 & 1.000 & -0.039 \\
\hline NLR & -0.124 & 0.066 & -0.090 & -0.134 & -0.121 & 0.190 & -0.031 & -0.039 & 1.000 \\
\hline
\end{tabular}

The aim of the article was to determine the association between neutrophil to lymphocyte-ratio (NLR), clinical disease severity and joint effusion in patients with early knee osteoarthritis. Studies have shown that NLR levels can be used as indicators of systemic inflammation supporting the inflammatory hypothesis of the pathogenesis of osteoarthritis [19]. A correlation between NLR levels, 
clinical severity and joint effusion in early osteoarthritis could be of use in developing new antiinflammatory treatments to slow down the progression.

In our analysis NLR was not associated with PROs or suprapatellar joint effusion. KOOSJR and age were the only independent predictors of NLR in the multivariate analysis. NLR was age dependent for both study subjects and controls.

Several studies found that age and NLR over 2.1 were predictive of advanced OA [11-14]. Tasoglu et al found that blood NLR $\geq 2.1$ had $50 \%$ sensitivity and $77 \%$ specificity in predicting severe knee $\mathrm{OA}$ and in the multivariate analysis, age and blood NLR $\geq 2.1$ were independent predictors of severe knee OA [11]. Mild to moderate OA was radiographically evaluated as Kellgren Lawrence stages 1 to 3 , and severe OA stage 4. However, for mild knee degeneration there is limited radiographic correlation. Furthermore, new MRI protocols have been able to determine changes in cartilage composition even before evident morphological signs [1,2].

There are several limitations of our study. The main one is the low number of subjects. Different associations may become apparent or more robust with large, multicentric cohorts. We hypothesized that joint effusion would be the most likely clinical factor to be associated with inflammatory markers such as NLR. It was measured using ultrasound and divided into 3 categories. The cutoff value of 4 $\mathrm{mm}$ was arbitrarily chosen, based on previously published data [4,5]. The normal interval for NLR has been reported in the range of 0.78-3.53, with an average of 1.65 [20]. Our control group has a higher female ratio but is still within the range at average of 1.98 .

Treatment options for early stages of OA are limited and evidence supporting them varies. Current options range from intraarticular instillations, filling of focal cartilage lesions to lower limb mechanical axis realignment. Rehabilitation procedures with stability and balance exercises may be applied for increasing mobility, strength and equilibrium [21]. A biomarker for early osteoarthritis highly corelated with clinical severity would be of great use for developing new treatments in early stages and for treatment follow up. Physical and chemical structure of joint have a great influence in disease progression and in the development of new types of treatments [22-24].

\section{Conclusions}

Neutrophil to lymphocyte ratio is within normal limits in patients with early stage knee osteoarthritis and is not associated with clinical severity or with joint effusion.

Acknowledgments: Andrei Ghiorghiţoiu, MD

\section{References}

1.HUNTER DJ, BIERMA-ZEINSTRA S. Osteoarthritis. Lancet 393, 2019, 1745-1759.

2.EMERY CA, WHITTAKER JL, MAHMOUDIAN A, et al. Establishing outcome measures in early knee osteoarthritis. , Nat Rev Rheumatol, 15,2019, 438-448.

3.NEES TA, ROSSHIRT N, ZHANG JA, et al., Synovial Cytokines Significantly Correlate with Osteoarthritis-Related Knee Pain and Disability: Inflammatory Mediators of Potential Clinical Relevance Clinic for Orthopedics and Trauma Surgery, J. Clin. Med., 8, 2019, E1343.

4.RIECKEBF, CHRISTENSEN R, TORP-PEDERSEN S, et al., An ultrasound score for knee osteoarthritis: a cross-sectional validation study, Osteoarthritis Cartilage, 22, 2014, 1675-91.

5.SAKELLARIOU G, CONAGHAN PG, ZHANG W, et al., EULAR recommendations for the use of imaging in the clinical management of peripheral joint osteoarthritis, Ann Rheum Dis., 76(9), 2017, 1484-1494.

6.WYATT FE, Osteoarthritis biomarkers: year in review, Osteoarthritis and Cartilage, 26, 2018, 312318.

7.ZHANG X, ZHANG W, FENG L-J, Prognostic Significance of Neutrophil Lymphocyte Ratio in Patients with Gastric Cancer: A Meta-Analysis, PLoS ONE, 9, 2014, e111906. 
8.MONTEIRO JÚNIOR JGM, TORRES DOC, DA SILVA MCFC, et al., Prognostic value of hematological parameters in patients with acute myocardial infarction: Intrahospital outcomes, PLoS One, 13(4),2018, e0194897.

9.ALEXANDRU L, HARAGUS H, DELEANU B, et al., Haematology panel biomarkers for humeral, femoral, and tibial diaphyseal fractures, Int Orthop., 43(7), 2019, 1567-1572. doi: 10.1007/s00264019-04305-1.

10.POP DV, VLAD DC, RADU D, et al., Lymphocyte and Neutrophil to Lymphocyte Ratio do not Predict Mortality in Hip Fracture Patients, Rev Chim., 70(8), 2019, 2854-2856

11.TAŞOĞLU Ö, BÖLÜK H, ŞAHIN ONAT Ş, et al., Is blood neutrophil-lymphocyte ratio an independent predictor of knee osteoarthritis severity?, Clin Rheumatol., 35, 2016, 1579-83.

12.CHANDRASHEKARA S, MUKHTAR AM, RENUKA P, et al., Characterization of neutrophil-tolymphocyte ratio as a measure of inflammation in rheumatoid arthritis, Int J Rheum Dis.,20, 2017, 1457-1467.

13.TAŞOĞLUÖ, ŞAHIN A, KARATAŞ G, et al., Blood mean platelet volume and platelet lymphocyte ratio as new predictors of hip osteoarthritis severity, Medicine (Baltimore), 96, 2017, e6073.

14.BÜYÜKAVCI R, AKTÜRK S, SAĞ S., Comparison of blood platelet distribution width and neutrophil-lymphocyte ratio in patients with different grades of knee osteoarthritis, $J$ Back Musculoskelet Rehabil., 31, 2018, 1035-1039.

15.TODOR A, CATEREV S, NISTOR DV., Outside-In Deep Medial Collateral Ligament Release During Arthroscopic Medial Meniscus Surgery, Arthrosc Tech., 5, 2016, e781-e785.

16.BEAUFILS P, BECKER R, KOPF S, et al., The knee meniscus: management of traumatic tears and degenerative lesions, EFORT Open Rev. 2017;2:195-203.

17.TODOR A, VERMESAN D, HARAGUS H, et al., Cross-cultural adaptation and validation of the Romanian International Knee Documentation Committee - subjective knee form, PeerJ, 8, 2020, e8448 https://doi.org/10.7717/peerj.8448.

18.FLORESCU S, VERMESAN D, HARAGUS H, et al., Cross-cultural adaptation and validation of the Romanian Knee disability and Osteoarthritis Outcome Score for Joint Replacement (KOOSJR), BMC Musculoskelet Disord. In review, 2020, DOI:10.21203/rs.2.18127/v2

19.HIRA S, TAMAM C. Diagnostic value of hematological parameters in patients with osteoarthritis, Cukurova Medical Journal. 42(1), 2017, 114-9.

20.FORGET P, KHALIFA C, DEFOUR JP, et al., What is the normal value of the neutrophil-tolymphocyte ratio?, BMC Res Notes., 10, 2017, 12.

21.ONOFREI RR, AMARICAI E, PETROMAN R, SUCIU O., Relative and absolute within-session reliability of the modified Star Excursion Balance Test in healthy elite athletes, PeerJ. 7, 2019, e6999.

22.SUCIU O, BERETEU L, DRĂGĂNESCU G, IOANOVICI T. Determination of the Elastic Modulus of Hydroxyapatite Doped with Magnezium through Nondestructive Testing. In Advanced Materials Research, Trans Tech Publications Ltd., 814, 2013, 115-122.

23.ANDOR B, PATRASCU JM, FLORESCU S, COJOCARU D, SANDESC M, BORCAN F, BORUGA O, BOLINTINEANU S. Comparison of different knee implants used on patients with osteoarthritis control study, Mater. Plast., 53(1), 2016, 119-125.

24.FODOR PAL, SOLYON A, FODOR R, et al., Role of the Biomimetic Scaffolds in the Regeneration of Articular Tissue in Deep Osteochondral Defects in a Rabbit Model, Rev. Chim., 69(1), 2018, 201-207

Manuscript received: 26.02 .2020 